Relations industrielles

Industrial Relations

\title{
La mobilité des travailleurs urbains, par Laurence C. Hunter et Graham L. Reid, O.C.D.E., Paris, 1968, 239 pp.
}

\section{Bernard Solasse}

Volume 23, numéro 4, 1968

URI : https://id.erudit.org/iderudit/027963ar

DOI : https://doi.org/10.7202/027963ar

Aller au sommaire du numéro

Éditeur(s)

Département des relations industrielles de l'Université Laval

ISSN

0034-379X (imprimé)

1703-8138 (numérique)

Découvrir la revue

Citer ce compte rendu

Solasse, B. (1968). Compte rendu de [La mobilité des travailleurs urbains, par Laurence C. Hunter et Graham L. Reid, O.C.D.E., Paris, 1968, 239 pp.] Relations industrielles / Industrial Relations, 23(4), 700-701.

https://doi.org/10.7202/027963ar

Tous droits réservés @ C Département des relations industrielles de l'Université Laval, 1968
Ce document est protégé par la loi sur le droit d'auteur. L'utilisation des services d'Érudit (y compris la reproduction) est assujettie à sa politique d'utilisation que vous pouvez consulter en ligne.

https://apropos.erudit.org/fr/usagers/politique-dutilisation/ 
- L'utilisation de l'espace urbain: ici l'auteur observe les comportements d'achat et les relations sociales qui demeurent ayant tout familiales. Les activités de loisirs ne sont pas envisagées comme moyens de promotion sociale: le but poursuivi demeure pour plusieurs la possession d'un confort familial. La mobilité géographique se limite ò la commune et au quartier.

Globalement l'étude montre que la tension entre deux modes de vie différents, l'un fondé sur les valeus traditionnelles d'entr'aide, de coopération; l'autre, fondé sur la consommation de masse, le profit, l'argent, n'a pas bouleversé l'ancien mode de vie à cause d'un manque de participotion des ouvriers dans le système.

\section{Claude CARBONNEAU}

L'emploi d temps partiel, Sixième rapport concernant l'emploi des groupes spéciaux, par Jean Hallaire, publié par la Direction de la Main-d'Oeuvre et des Affaires Sociales de I'O.C.D.E., Paris, 1968, 118 pp.

L'emploi à temps partiel o pris dans de nombreux pays de plus en plus d'importance. II répond aux besoins des travailleurs qui ne veulent ou ne peuvent travailler à plein temps.

L'auteur examine divers groupes de personnes pour lesquelles un emploi régulier à temps partiel semble approprié: les hommes qui ont des responsabilités familiales, les handicapés physiques, les étudiants, les personnes âgées, les veuves et divorcées et les femmes mariées. L'analyse repose sur l'expérience acquise dans six pays: Royaume-Uni, Allemagne, Suède, Danemark, Belgique, France, Etats-Unis et le Conada.

L'auteur passe ensuite en revue les arguments économiques et socioux en faveur du travail à temps partiel ainsi que ceux avancés por les organisations d'employeurs et de trovail contre l'emploi à temps partiel.

Les obstacles à l'emploi à temps partiel tels que la pression fiscale, l'inégalité des solaires entre hommes et femmes, et d'autres, sont analysés et des suggestions sont offertes pour les surmonter. Les garanties conventionnelles et réglementaires nécessaires à une meilleure intégration de ce type d'emploi dans le marché du travail sont étudiées.

Ce ropport fait des prévisions quant ò la place future de l'emploi à temps partiel, notamment pour les groupes d'activités suivants: infirmières, secteur public et paro- public (I'enseignement), l'industrie, le secteur tertiaire: en particulier les octivités de loisirs.

Les conclusions et recommandations de l'outeur intéresseront tous les services gouvernementaux, les associations d'employeurs et de travoilleurs qui cherchent des solutions aux problèmes soulevés par l'emploi d temps partiel.

\section{Pierre DIONNE}

La mobilité des travailleurs urbains, par Laurence C. Hunter et Graham L. Reid, O.C.D.E., Paris, 1968, 239 pp.

Cette étude complète la liste des publications approuvées par le comité de la maind'oeuvre et des affaires sociales de l'organisation de coopération et de développement économiques, dont l'intérêt justifie que nous roppelions ici les titres:

$H$. KRIER, Main-d'oeurre rurale et le développement industriel, O.C.D.E.

G. BARBICHON, Adaptation ef formation de la main-d'oeurre des régions rurales au travail industriel, O.C.D.E.

G. BEIJER, La main-d'oeuvre rurale nationale, son adaptation à l'industrie, O.C.D.E

R. DESCLOITRES, Le travailleur étranger ; son adaptation au travail industriel et ò la vie urbaine, O.C.D.E.

Dons leur étude MM. Hunter et Reid érigent en principe que la ropidité du progrès technologique et, son corrélat, l'évolution des structures de l'emploi exigent un occroissement de la mobilité de la main-d'oeuvre. Ce raisonnement les conduit ò légitimer une intervention des pouvoirs publics destinée d̀ inciter les travailleurs à délaisser volontairement les régions et les industries en déclin au profit de régions et $d$ 'industries en expansion.

Certes ces auteurs n'ignorent pas les facteurs de résistance "non économiques * propres à freiner la mobilité géographique des travailleurs, mais semblent les sousestimer quelque peu. C'est là l'une des limites logiques d'une opproche trop exclusivement d'ordre économique.

Les conclusions, qui ne sont en fait que des recommandations à peine voilées, nous paraissent trop générales pour pouvoir servir de base à l'élaboration de politiques gouvernementales effectives, tant il est vrai qu'en cette matière, les motivotions et les décisions des individus dans un contexte et des situa- 
::ons vécues où les contraintes culturelles, familiales, sociologiques, s'inscrivent souvent à l'encontre des conclusions d'un calcul fondé sur une rationnalité purement économique. Quoiqu'il en soit, I'on ne saurait établir des politiques gouvernementales efficaces en cette matière sans tenir compte des données spécifiques d'ordre national et régional.

Nous voudrions souligner, en conclusion, la valeur pédagogique de cette étude; ellé tient surtout à la clarté avec laquelle les auteurs définissent le sens des concepts qu'ils emploient. Tout en excluont toute simplificotion abusive.

Une importante bibliographie, deux cent treize livres ou articles de revue, complète cet exposé qui devient un instrument de trovail précieux pour l'étudiant, le professeur et le chercheur.

\section{Bernard SOLASSE}

Réflexions d'un citoyen sur l'avenir du Québec - sur quelques aspects de l'expérience suédoise, par Jean-Paul Lefevre, Cahiers Cité Libre, Editions du Jour, Ottowa, 1968, 120 pages.

Le premier thème, politique, et d'une actualité brûlante, n'appelle guère de commentaires dans cette revue consacrée aux relations industrielles. Jean-Paul Lefèvre est député libéral à Québec, il entend prendre ses distances par rapport aux thèses développées par René Lévesque dans son livre Option Québec. Sur ce point le lecteur jugero comme citoyen.

Le second thème, la seconde partie, est une excellente étude de style journalistique sur quelques aspects de l'expérience suédoise * dont la qualité tranche par rapport à la pauvreté de bien des écrits de journalistes de métier consacrés aux questions internationales ou étrangères.

Jean-Paul Lefèvre n'est animé par aucune prétention a scientifique * et, se souvenant de son passé de syndicaliste, il s'intéresse et s'interroge notamment sur le système de relations industrielles suédois, non sans se départir d'un esprit critique qui le conduit ¿ ne pas ériger en modèle universel les pratiques existant en Suède.
On the Theory of Socialist Planning, by J.G. ZIELINSKI, Oxford University Press, Ibadan, 1968, 170 pp.

Voilà un ouvrage construit sur la base théorique d'une série de cours donnés par l'auteur ò * The Nigerian Institute of Social and Economic Research ». Le contenu même des cours retient les résultats d'une étude de l'économie socialiste, étude entreprise par un groupe d'économistes polonais.

Globalement, le lecteur nord-américain de cet ouvrage retrouvera posés les grands principes de la planification économique, ses postulats et conditions. II retrouvera aussi les grands problèmes contemporains au niveau de l'application et de l'exécution d'un plon national; signalons seulement l'efficacité des investissements, le gaspillage des ressources, la centralisation et la décentralisation des décisions, l'adéquation de la consommation aux lois du développement, etc., etc.

Mais cet ouvrage contient plus que cela puisqu'il cherche à définir une théorie de la planification socialiste. Le lecteur est donc saisi par des problèmes particuliers des mécanismes et des instruments différents, des principes et des lois distinctes. Ce sont les problèmes de la création des marchés, de la programmation des prix; les principes relatifs à la nationalisation des secteurs et unités économiques stratégiques; les moyens de calculer et d'évaluer la production, la consommation, les investissements à une échelle globale.

Dans l'optique particulière de la planification socialiste, l'auteur centre la présentation des études sur une méthode utilisée par les planificateurs socialistes, le a direct economic calculation ». C'est précisément cette méthode qu'il développe, méthode qui vise à donner une description théorique des économies socialistes existantes. La méthode est liée plus précisément au niveau opérationnel des plans, car elle vise à donner des techniques de calcul et de description des plans dont les grands objectifs concernent la partie prospective de ces plans.

On retrouve avec intérêt des préoccupations que nous partageons au niveau de la planification nationale; on découvre des techniques et des instruments nouveaux qui pourraient peut être nous servir, même en régime néo-capitoliste. 